OPEN ACCESS

Edited by:

Marjolein Bonthuis,

Academic Medical

Center, Netherlands

Reviewed by:

Timo Jahnukainen,

New Children's Hospital, Helsink

University Hospital, Finland

Evgenia Preka,

Southampton Children's Hospital,

United Kingdom

*Correspondence:

Rahul Chanchlan

chanchlr@mcmaster.ca

Specialty section:

This article was submitted to

Pediatric Nephrology,

a section of the journal

Frontiers in Pediatrics

Received: 16 July 2021 Accepted: 03 September 2021

Published: 27 October 2021

Citation:

Ulrich EH, So G, Zappitelli M and Chanchlani R (2021) A Review on the

Application and Limitations of

Administrative Health Care Data for

the Study of Acute Kidney Injury

Epidemiology and Outcomes in

Children. Front. Pediatr. 9:742888.

doi: $10.3389 /$ fped.2021.742888

\section{A Review on the Application and Limitations of Administrative Health Care Data for the Study of Acute Kidney Injury Epidemiology and Outcomes in Children}

\author{
Emma H. Ulrich ${ }^{1}$, Gina So ${ }^{2}$, Michael Zappitelli ${ }^{3}$ and Rahul Chanchlani ${ }^{4,5,6 *}$ \\ ${ }^{1}$ Division of Pediatric Nephrology, Department of Pediatrics, University of Alberta, Edmonton, AB, Canada, ${ }^{2}$ Department of \\ Health Sciences, McMaster University, Hamilton, ON, Canada, ${ }^{3}$ Division of Nephrology, Department of Pediatrics, Hospital \\ for Sick Children, University of Toronto, Toronto, ON, Canada, ${ }^{4}$ Institute of Clinical and Evaluative Sciences, Ontario, ON, \\ Canada, ${ }^{5}$ Department of Health Research Methods, Evidence and Impact, McMaster University, Hamilton, ON, Canada, \\ ${ }^{6}$ Division of Pediatric Nephrology, Department of Pediatrics, McMaster University, Hamilton, ON, Canada
}

Administrative health care databases contain valuable patient information generated by health care encounters. These "big data" repositories have been increasingly used in epidemiological health research internationally in recent years as they are easily accessible and cost-efficient and cover large populations for long periods. Despite these beneficial characteristics, it is also important to consider the limitations that administrative health research presents, such as issues related to data incompleteness and the limited sensitivity of the variables. These barriers potentially lead to unwanted biases and pose threats to the validity of the research being conducted. In this review, we discuss the effectiveness of health administrative data in understanding the epidemiology of and outcomes after acute kidney injury (AKI) among adults and children. In addition, we describe various validation studies of AKI diagnostic or procedural codes among adults and children. These studies reveal challenges of AKI research using administrative data and the lack of this type of research in children and other subpopulations. Additional pediatric-specific validation studies of administrative health data are needed to promote higher volume and increased validity of this type of research in pediatric AKI, to elucidate the large-scale epidemiology and patient and health systems impacts of AKI in children, and to devise and monitor programs to improve clinical outcomes and process of care.

Keywords: administrative health care data, acute kidney injury, nephrology, epidemiology, pediatrics

\section{INTRODUCTION}

Acute kidney injury (AKI), characterized by an abrupt deterioration of kidney function, is common in adults and children, and the incidence is increasing (1). The Kidney Disease Improving Global Outcomes (KDIGO) definition incorporates rise in serum creatinine and decreased urine output to identify AKI and classify severity. Approximately 5\% of hospitalized children develop AKI (2), and $20-50 \%$ of children in the intensive care unit (ICU) and those with cardiac surgery develop AKI $(3,4)$. Pediatric and adult AKIs are associated with adverse in-hospital outcomes, including 
mortality and prolonged hospitalization. Many studies among adults have shown that AKI is a strong risk factor for chronic kidney disease (CKD), end-stage kidney disease (ESKD), hypertension (HTN), cardiovascular disease, and mortality (5). Among children, available data suggest that AKI is also associated with worse long-term outcomes (6-9).

Administrative health care databases have enabled expansive observational studies because these data are pre-collected and easily accessible and provide a wealth of information regarding epidemiology, risk factors, and outcomes of AKI (10). The purpose of this review is to provide an overview of administrative health care data, as well as its strengths and limitations. In addition, we review validation studies to identify individuals with AKI using administrative health data. Finally, we provide an update on the incidence, risk factors, and outcomes after AKI, as learned from the studies conducted in the last 5 years using administrative health data.

\section{ADMINISTRATIVE HEALTH CARE DATA RESEARCH}

Administrative health care databases store large quantities of information that are routinely compiled and updated by provinces and/or countries on patients, health care providers, and institutions during various patient encounters $(11,12)$. Examples of data collected are summarized in Figure 1 and include physician billing codes, prescription claims, vital records, and hospitalization/discharge summaries (13). These data contained within the administrative health databases are often referred to as "big data," which are distinguished by the large volume of information, speed at which it is generated, and the wide range of fields that it covers (14). Epidemiological studies, in particular, benefit greatly from the availability of administrative health care databases to evaluate and track the health of large populations over a period (12). For example, the International Classification of Diseases (ICD) diagnostic codes found in the databases have been established as an international standard for classification of diseases $(15,16)$. Using standardized ICD diagnostic codes as a main source of data allows clinical epidemiologists to study patterns of disease, patient care, and various health outcomes in an efficient way (17).

In recent years, the use of administrative health data as a powerful research tool has become increasingly established and extensively applied to various fields of epidemiological research $(11,15,16)$. AKI, in particular, is an ideal disease to study using administrative health data (18) due to the high prevalence in hospitalized patients, increased risk for major morbidity (CKD, progression to ESKD, and death), financial costs, and recent research demonstrating that care of patients with AKI is suboptimal due to avoidable systemic issues (18). A series of statements from the Acute Dialysis Quality Initiative (ADQI) consensus conference in 2015 outline the potential of large database research for AKI, including real-time prediction of risk for AKI, developing electronic alerts for quality improvement, and "tagging" AKI patients for longitudinal care and advancing our understanding of long-term outcomes following AKI (1823). Currently, real-time AKI alerts using electronic systems notify health care providers using the KDIGO definition of AKI events (20). In the future, however, machine learning could incorporate real-time patient comorbidities, nephrotoxin exposure, and so on that would allow these electronic systems to provide prognostic data directly to health care providers managing the individual patient, even before an AKI event has occurred (20). The potential real-time application of "big data" to the management of AKI at the individual, organizational, and institutional level is summarized in Figure 2.

\section{STRENGTHS AND LIMITATIONS OF ADMINISTRATIVE HEALTH DATA RESEARCH}

To effectively conduct and interpret outcomes of administrative health data research, it is important to understand the strengths and limitations (Table 1).

\section{Strengths}

One of the most significant advantages of administrative health care data is the very large sample size, which in turn reduces sampling error, increases statistical power, and increases validity $(16,24)$. The inclusion of a larger and more diverse population also increases generalizability of the study findings to a greater population (25). Having larger numbers of variables collected also allows researchers adjust for many types of variables in multivariable analyses (24).

Although prospective randomized controlled trials (RCTs) are considered to be the gold standard of scientific evidence in clinical medicine, it is often difficult to conduct them because of various logistical, financial, and ethical limitations (24). As administrative health care data are pre-collected and readily available, they allow studies to be less costly and much quicker to perform (24). In fact, cluster RCTs that obtain data through administrative health databases can act as a solution to allow for inclusion of a broad number of facilities and larger representative samples of patients (26). As with any cluster RCT, care would be required to ensure information about exposure status, and outcomes are generalizable and derived from data sources in a consistent manner across trial centers. The heterogeneity and accessibility of administrative health data also allow for easier identification of patients with rare diseases (27-29).

Administrative health care databases often allow patients to be followed over extended time periods $(6,30,31)$. Unlike studies that require primary data collection, administrative health databases do not involve researchers having to contact individuals, and loss to follow-up is not a significant issue (unless the patients do not immigrate) when conducting longitudinal studies for outcomes. In fact, researchers can follow patients over a long period by linking their clinical research records to administrative databases with the patients' consent (32). This, in turn, simplifies the follow-up process and eliminates any chances of non-response and recall bias (33). 


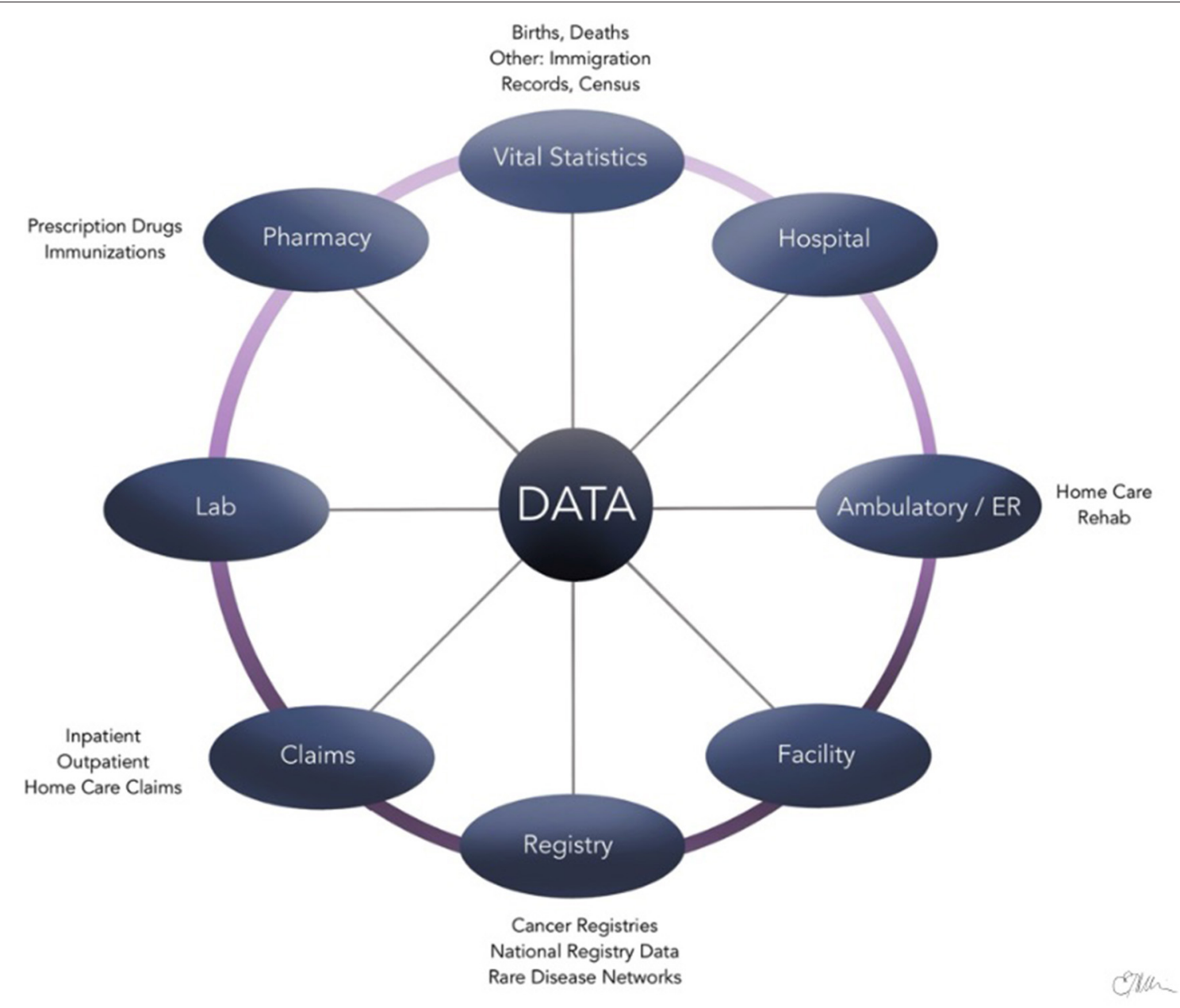

FIGURE 1 | Sources of administrative health data.

Administrative health care data also can be supplemented and linked with other data sources, allowing for a larger and more comprehensive data set. This involves the easily computerized process of linking an individual using personal identifier variables across different databases without affecting patient privacy (16). Other data sources, such as census or citizenship data, allow researchers to gain more information about certain target groups' socioeconomic status and lifestyle $(34,35)$. Linkage with electronic medical records, which includes more detailed information about a patient's health, helps to increase the validity of research findings $(36,37)$.

\section{Limitations}

Because of health care databases being structured primarily for administrative and billing purposes, they often lack additional clinical information about the diseases of interest, health outcomes, and medications (38). Detailed data about some comorbidities, anthropometric measures, quality of life, education status, physical activity levels, and patient-reported outcomes are usually unavailable or recorded inconsistently and can negatively impact the research process (39-41). Additionally, changes in clinical diagnostic billing coding practices and different software systems that different physicians and institutions use may cause additional gaps in data (41). For example, the mandatory transition from ICD-9 to ICD-10 diagnostic codes in 2015 involved nearly four times more codes (42). Hence, it is important to consider the changing thresholds and other factors for diagnosing certain diseases to prevent overestimation or underestimation of cases when evaluating incidence and outcomes, which is mainly accomplished through regular and rigorous validation studies. To address this, it is essential for policymakers to devise a standard framework surrounding the coding and software system used within institutions to help bridge the data and communication gaps. This will help ensure that the collection of data is more consistent within and between countries and that the information can be used more effectively in research studies.

Patient identification in administrative health data research is usually made upon the basis of specific diagnostic or procedural codes of disease (11). Validation studies that evaluate the different 


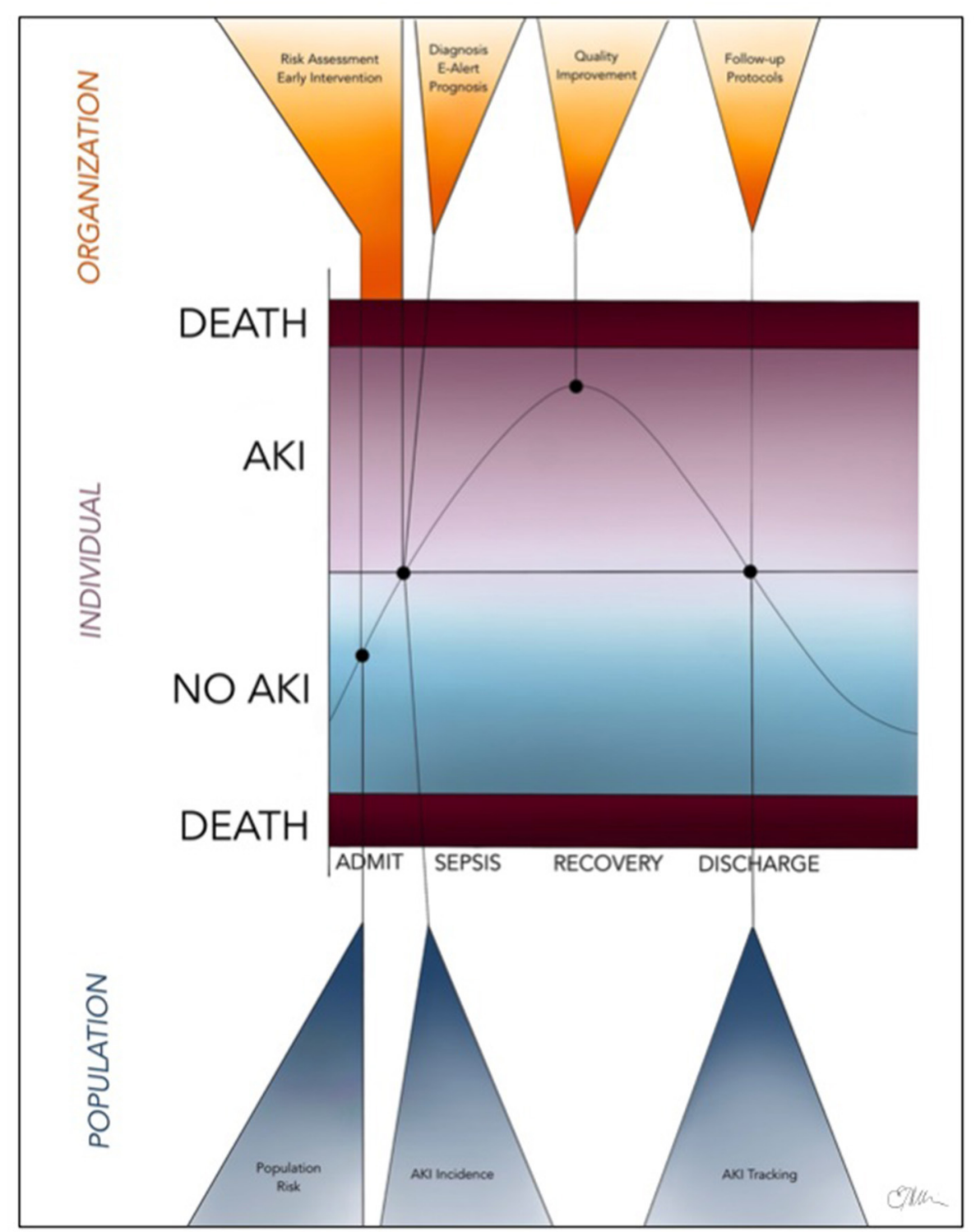

FIGURE 2 | Future, real-time application of "big data" (including administrative health data and electronic medical records) to the identification, management, and monitoring of children and adults with AKI at the individual, organizational, and population levels.

combination of codes and criteria, also known as "algorithms," are essential when conducting administrative health research, but are currently very limited $(11,43)$. A scoping review for validation studies of administrative health data from 2014 was able to identify only a very limited number of pediatric validation studies for a wide range of diseases and revealed that most studies focused exclusively on adult populations (44).

When working with larger sample sizes in administrative data research, issues related to statistical analyses may occur. For instance, although associations between outcome variables and exposures present as statistically significant $(p<0.05)$ due to a large sample size and power, clinical significance is not always evident and should be critically evaluated when reviewing the results (25). The clustered nature of the data sets in administrative health databases may also result in overestimating associations, which may lead to incorrect conclusions, and ideally should be addressed through models that take into account the hierarchical nature of data present (11).

Just like any other type of study, administrative health data research has the potential to be affected by information bias. Misclassification of an outcome can result when there is unclear or unavailable clinical documentation or a range of definitions for a certain disease, as mentioned previously (16). To address this, adding additional data sources through data linkage can be performed (16). Confounding by indication (also known as indication bias) commonly occurs when people with certain 
TABLE 1 | Summary of strengths and limitations of administrative health research.

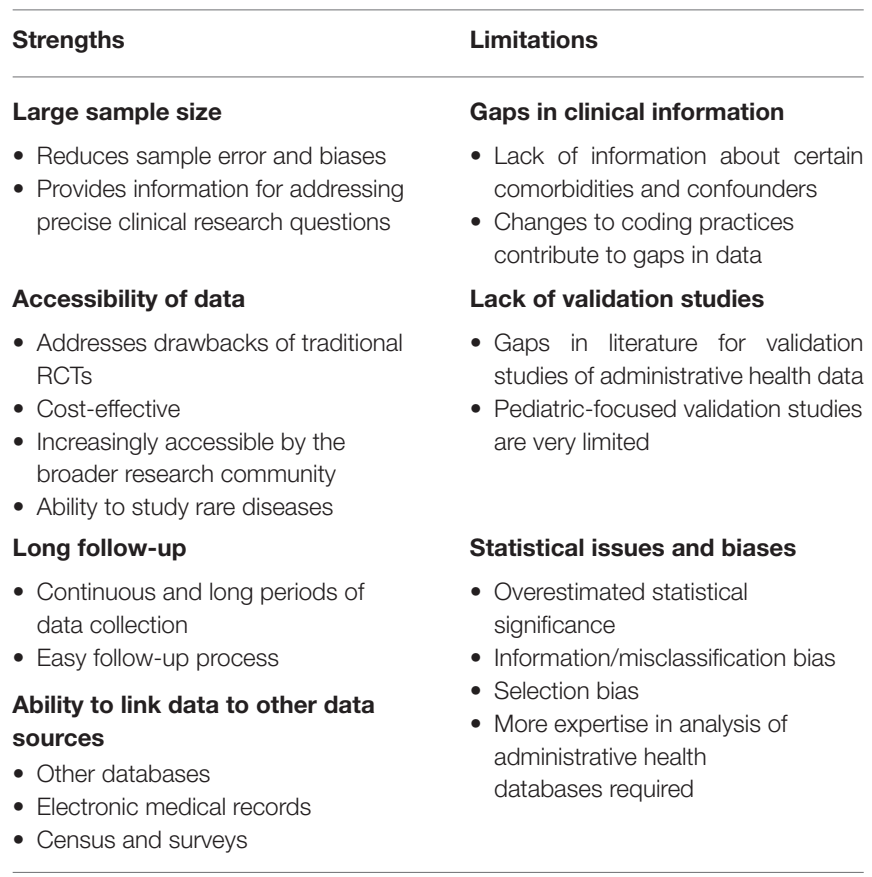

conditions are more likely to get certain tests or diagnoses attributed to them (45). For example, if someone already has AKI, they may be more likely to be diagnosed with CKD, but this does not necessarily mean they were originally at a higher risk for having CKD.

Additionally, selection bias may result when certain diagnostic codes do not selectively represent a condition under study as study samples will be restricted to a special population, especially when consent is required (11). Careful planning of studies should be done to avoid these biases. When carrying out different objectives during an administrative health data-based research study, using a multidisciplinary team of researchers that include statisticians with expertise in health data analysis and modeling of the specific database being used is essential $(11,39)$.

\section{VALIDATION STUDIES FOR ADMINISTRATIVE HEALTH CARE DATA IN AKI}

Since the late 1990s, there has been increasing interest in validating the use of discharge and procedural billing codes from administrative health care data research on AKI, including severe AKI requiring kidney replacement therapy (KRT) (46). In 2011, Vlasschaert et al. published a systematic review on the validity of using administrative codes for AKI and CKD (47). The results of this review showed high specificity but lower sensitivity for identifying AKI using administrative data; of note, sensitivity is improved when administrative health care data are used to identify more severe AKI and/or AKI treated with KRT. We conducted a MEDLINE search with the following search terms:
(1) acute kidney injury, aki, acute renal failure, or continuous renal replacement therapy, and (2) admin* adj3 data* to identify validation studies published since this systematic review, which identified an additional eight studies. A summary of the results of these studies (48-55) and a selection of those published in the systematic review (56-61) are included in Table 2. To our knowledge, this represents the most up-to-date summary of validation studies for AKI administrative health research.

We have summarized 14 studies that took place between 2004 and 2020. Validation studies have generally compared cases of AKI identified from administrative data, including diagnostic and/or procedure codes for AKI, to a reference standard. More recent validation studies tended to use $I C D-10$ codes $(50,52,55$, $59)$, although many studies utilized ICD-9 codes (51, 53, 54, 56$61)$. For the most part, the reference standard has consisted of either standardized chart review $(48,50-53,56,57,59)$ or laboratory data linked to either an (1) electronic health record $(49,51)$ or (2) administrative database containing data from inpatient and outpatient laboratories $(54,55,58,60,61)$. The source for the reference standard significantly impacted study size with full chart reviews tending to have much smaller study populations. Early studies used acute renal failure (ARF) (5661) for the reference standard, but the definition of ARF was not always well-defined. Newer studies have tended toward using KDIGO criteria (62) for AKI using serum creatinine levels as the reference standard $(50,51,53)$; two studies used serum creatinine or urine output criteria $(51,53)$. Several studies compared only patients with more severe AKI against the reference standard (48, $51,53,55)$. Most studies were done in hospitalized adults $(48,50-$ 52, 54-61); two studies examined adults admitted to the ICU $(48,55)$, and two early studies examined adults post-coronary artery bypass graft (58) operation and post-acute myocardial infarction (59). Only two studies were done in children $(49,53)$; one study examined hospitalized pediatric patients exposed to nephrotoxins (49) and the other examined pediatric patients admitted to the ICU (53).

Most studies reported various parameters, such as sensitivity, specificity, positive predictive value (PPV), and negative predictive value (NPV) of the administrative data vs. reference data for identifying cases of AKI or ARF. Specificity of administrative data tended to be high (86.7-100\%). Studies comparing administrative data to a standardized chart review, regardless of definition used, had a high specificity ( $\geq 94 \%)$ (48$53,56,57,59-61$ ). Two studies compared administrative data to another administrative database and reported reduced specificity (86.7-98.3\%); however, these results are likely to be impacted by the quality of data in the administrative database used as a reference standard and are less reliable. However, sensitivity tended to be highly variable (5.9-94.5\%). Studies with higher severity of AKI or AKI receiving KRT or ARF generally reported a higher sensitivity. When compared to standardized chart review as the reference standard $(51,53)$, administrative data identified patients with stage 3 AKI by KDIGO criteria with a sensitivity of $36.5 \%$ in hospitalized adults (51), $14.3 \%$ in pediatric cardiac surgery patients in ICU (53), and $42.2 \%$ for pediatric non-cardiac surgery patients in ICU (53). In one study of billing codes of adult hospitalizations, applying criteria for creatinine or urine output 
TABLE 2 | Summary of validation studies for acute kidney injury and renal replacement therapy codes in adults and pediatric administrative health research.

\begin{tabular}{|c|c|c|c|c|c|c|c|c|c|c|c|}
\hline References & $\begin{array}{l}\text { Validated } \\
\text { Measure }\end{array}$ & $\begin{array}{l}\text { Study } \\
\text { Population }\end{array}$ & $\mathbf{N}$ & $\begin{array}{l}\text { Administrative } \\
\text { Health Data } \\
\text { Source }\end{array}$ & $\begin{array}{l}\text { Reference } \\
\text { Standard }\end{array}$ & & & Study Findings & & & Limitations \\
\hline \multirow[t]{3}{*}{$\begin{array}{l}\text { Quan et al. } \\
\text { (56) }\end{array}$} & \multirow[t]{3}{*}{ ARF } & \multirow{3}{*}{$\begin{array}{l}\text { Adults } \\
\text { hospitalized in } \\
\text { Canada in } \\
\text { 1996-1997 }\end{array}$} & \multirow{3}{*}{$\begin{array}{l}1,200 \text { (600 } \\
\text { medical service, } \\
600 \text { general } \\
\text { surgical service) }\end{array}$} & \multirow{3}{*}{$\begin{array}{l}\text { Discharge billing } \\
\text { codes, including ICD-9 } \\
\text { diagnostic codes for } \\
\text { ARF }\end{array}$} & \multirow[t]{3}{*}{$\begin{array}{l}\text { Standardized review of } \\
\text { medical charts for ARF }\end{array}$} & & $\begin{array}{l}\text { Sensitivity-\% } \\
\text { (Cl) }\end{array}$ & $\begin{array}{l}\text { Specificity-\% } \\
\text { (CI) }\end{array}$ & PPV-\% (CI) & NPV-\% (Cl) & \multirow{3}{*}{$\begin{array}{l}\text { - Small sample size } \\
\text { may } \\
\text { limit generalizability } \\
\text { - Reference standard } \\
\text { definition of ARF } \\
\text { is unclear } \\
\text { - Cohort admitted in } \\
\text { 1996-1997; higher } \\
\text { sensitivity may be } \\
\text { apparent based on } \\
\text { more current practices } \\
\text { and increased } \\
\text { AKI awareness }\end{array}$} \\
\hline & & & & & & ARF & 33.3 (Cl NR) & 99.1 (CI NR) & 15.4 (CI NR) & 99.7 (Cl NR) & \\
\hline & & & & & & & & & & & \\
\hline \multirow[t]{3}{*}{$\begin{array}{l}\text { Liangos et al. } \\
(57)\end{array}$} & \multirow[t]{3}{*}{ ARF } & \multirow{3}{*}{$\begin{array}{l}\text { Adults without } \\
\text { ESRD } \\
\text { hospitalized in } \\
\text { USA in } 2001\end{array}$} & \multirow[t]{3}{*}{13,237} & \multirow{3}{*}{$\begin{array}{l}\text { NHDS administrative } \\
\text { database, which } \\
\text { provides discharge } \\
\text { billing codes, including } \\
\text { ICD-9 diagnostic and } \\
\text { procedure codes for } \\
\text { ARF }\end{array}$} & \multirow{3}{*}{$\begin{array}{l}\text { Standardized review of } \\
\text { medical charts with } \\
\text { ARF defined using } \\
\text { variable change } \\
(104,105)\end{array}$} & & $\begin{array}{l}\text { Sensitivity-\% } \\
\text { (CI) }\end{array}$ & $\begin{array}{l}\text { Specificity-\% } \\
\text { (CI) }\end{array}$ & PPV-\% (CI) & NPV-\% (Cl) & \multirow{3}{*}{$\begin{array}{l}\text { - Cohort admitted in } \\
\text { 2001; higher sensitivity } \\
\text { may be apparent } \\
\text { based on more current } \\
\text { practices and } \\
\text { increased } \\
\text { AKI awareness } \\
\text { - Validation study done } \\
\text { in single tertiary centre } \\
\text { which may } \\
\text { limit generalizability }\end{array}$} \\
\hline & & & & & & ARF & 19.2 (Cl NR) & 99.6 (Cl NR) & 87.6 (CI NR) & 90.1 (Cl NR) & \\
\hline & & & & & & & & & & & \\
\hline \multirow[t]{3}{*}{$\begin{array}{l}\text { Parker et al. } \\
(58)\end{array}$} & \multirow[t]{3}{*}{ ARF } & \multirow{3}{*}{\begin{tabular}{|l} 
Adults \\
hospitalized for \\
CABG in USA \\
from 2000 to \\
2001
\end{tabular}} & \multirow[t]{3}{*}{38,230} & \multirow[t]{3}{*}{$\begin{array}{l}\text { Discharge billing codes } \\
\text { using ICD-9 diagnostic } \\
\text { codes for ARF }\end{array}$} & \multirow{3}{*}{$\begin{array}{l}\text { CABG Mortality } \\
\text { Reporting Program, } \\
\text { which provides clinical } \\
\text { registry data, including } \\
\text { complications }\end{array}$} & & $\begin{array}{l}\text { Sensitivity-\% } \\
\text { (Cl) }\end{array}$ & $\begin{array}{l}\text { Specificity-\% } \\
\text { (Cl) }\end{array}$ & PPV-\% (CI) & NPV-\% (Cl) & \multirow{3}{*}{$\begin{array}{l}\text { - CABG cohort may } \\
\text { limit generalizability } \\
\text { - Absence of a "gold } \\
\text { standard," Reference } \\
\text { standard did not use } \\
\text { chart reviewed data } \\
\text { and definition of ARF } \\
\text { is unclear } \\
\text { - Cohort admitted in } \\
\text { 2000-2001; higher } \\
\text { sensitivity may be } \\
\text { apparent based on } \\
\text { more current practices } \\
\text { and increased } \\
\text { AKI awareness }\end{array}$} \\
\hline & & & & & & ARF & 22.4 (Cl NR) & 98.3 (Cl NR) & 31.7 (CI NR) & 97.3 (CI NR) & \\
\hline & & & & & & & & & & & \\
\hline
\end{tabular}


TABLE 2 | Continued

\begin{tabular}{|c|c|c|c|c|c|c|c|c|c|c|c|}
\hline References & $\begin{array}{l}\text { Validated } \\
\text { Measure }\end{array}$ & $\begin{array}{l}\text { Study } \\
\text { Population }\end{array}$ & $\mathbf{N}$ & $\begin{array}{l}\text { Administrative } \\
\text { Health Data } \\
\text { Source }\end{array}$ & $\begin{array}{l}\text { Reference } \\
\text { Standard }\end{array}$ & & & Study Findings & & & Limitations \\
\hline \multirow[t]{4}{*}{ So et al. (59) } & \multirow[t]{4}{*}{ ARF } & \multirow{4}{*}{$\begin{array}{l}\text { Adults } \\
\text { hospitalized } \\
\text { with AMI in } \\
\text { Canada from } \\
1994 \text { to } 2004\end{array}$} & \multirow[t]{4}{*}{193} & \multirow{4}{*}{$\begin{array}{l}\text { Discharge billing codes } \\
\text { using either ICD-9 } \\
\text { (from } 1994 \text { to 2000) } \\
\text { and ICD-10 (from 2001 } \\
\text { to 2004) diagnostic and } \\
\text { procedure codes for } \\
\text { ARF }\end{array}$} & \multirow[t]{4}{*}{$\begin{array}{l}\text { Standardized review of } \\
\text { medical charts for ARF }\end{array}$} & & $\begin{array}{l}\text { Sensitivity-\% } \\
\text { (CI) }\end{array}$ & $\begin{array}{l}\text { Specificity-\% } \\
\text { (CI) }\end{array}$ & PPV-\% (Cl) & NPV-\% (Cl) & \multirow{4}{*}{$\begin{array}{l}\text { - Small sample size, } \\
\text { cohort with AMI may } \\
\text { limit generalizability } \\
\text { - Reference standard } \\
\text { was collected in a } \\
\text { different province and } \\
\text { definition of ARF } \\
\text { is unclear } \\
\text { - Cohort admitted in } \\
\text { 1994-2004; higher } \\
\text { sensitivity may be } \\
\text { apparent based on } \\
\text { more current practices } \\
\text { and increased } \\
\text { AKl awareness }\end{array}$} \\
\hline & & & & & & \begin{tabular}{|l} 
ARF \\
$($ ICD-10, \\
$2001-2004)$
\end{tabular} & $\begin{array}{l}80.0 \\
(51.91-95.67)\end{array}$ & \begin{tabular}{|l}
95.5 \\
$(91.34-98.04)$
\end{tabular} & $\begin{array}{l}60.0(36.05- \\
80.88)\end{array}$ & $\begin{array}{l}98.3 \\
(95.02-99.64)\end{array}$ & \\
\hline & & & & & & $\begin{array}{l}\text { ARF (ICD-9, } \\
\text { 1994-2000) }\end{array}$ & $\begin{array}{l}80.0 \\
(51.91-95.67)\end{array}$ & $\begin{array}{l}98.3 \\
(95.15-99.65)\end{array}$ & $\begin{array}{l}80.0(51.91- \\
95.67)\end{array}$ & $\begin{array}{l}98.3 \\
(95.15-99.65)\end{array}$ & \\
\hline & & & & & & & & & & & \\
\hline \multirow[t]{4}{*}{$\begin{array}{l}\text { Waikar et al. } \\
(60)\end{array}$} & \multirow[t]{4}{*}{ ARF } & \multirow{4}{*}{\begin{tabular}{|l} 
Adults \\
hospitalized in \\
USA in 1994 \\
and 2002
\end{tabular}} & \multirow{4}{*}{$\begin{array}{l}26,751 \\
\text { admissions } \\
(19,206 \text { in 2002; } \\
7,545 \text { in 1994) }\end{array}$} & \multirow{4}{*}{$\begin{array}{l}\text { NIS administrative } \\
\text { database, which } \\
\text { provides discharge } \\
\text { billing codes, including } \\
\text { ICD-9 diagnostic and } \\
\text { procedure codes for } \\
\text { ARF }\end{array}$} & \multirow{4}{*}{$\begin{array}{l}\text { Linked laboratory and } \\
\text { hospital administrative } \\
\text { data with ARF defined } \\
\text { as } 100 \% \text { increase } \\
\text { between nadir and } \\
\text { peak SCr }\end{array}$} & & $\begin{array}{l}\text { Sensitivity-\% } \\
\text { (Cl) }\end{array}$ & $\begin{array}{l}\text { Specificity-\% } \\
\text { (CI) }\end{array}$ & PPV-\% (Cl) & NPV-\% (Cl) & \multirow{4}{*}{$\begin{array}{l}\text { - Reference standard } \\
\text { based on laboratory } \\
\text { data may have resulted } \\
\text { in misclassification of } \\
\text { ARF as } \\
\text { diagnostic standard } \\
\text { - Cohort admitted in } \\
1994 \text { and 2002; higher } \\
\text { sensitivity may be } \\
\text { apparent based on } \\
\text { more current practices } \\
\text { and increased } \\
\text { AKl awareness } \\
\text { - Validation study done } \\
\text { in two academic } \\
\text { centres, which may } \\
\text { limit generalizability }\end{array}$} \\
\hline & & & & & & ARF (2002) & 29.3 (CI NR) & 97.4 (Cl NR) & 59.1 (Cl NR) & 91.5 (CI NR) & \\
\hline & & & & & & ARF (1994) & 17.4 (Cl NR) & 98.7 (CI NR) & $63.5(\mathrm{Cl} N R)$ & 89.9 (CI NR) & \\
\hline & & & & & & & & & & & \\
\hline
\end{tabular}


TABLE 2 | Continued

\begin{tabular}{|c|c|c|c|c|c|c|c|c|c|c|c|}
\hline References & $\begin{array}{l}\text { Validated } \\
\text { Measure }\end{array}$ & \begin{tabular}{|l} 
Study \\
Population
\end{tabular} & $\mathbf{N}$ & $\begin{array}{l}\text { Administrative } \\
\text { Health Data } \\
\text { Source }\end{array}$ & $\begin{array}{l}\text { Reference } \\
\text { Standard }\end{array}$ & & & Study Findings & & & Limitations \\
\hline \multirow[t]{4}{*}{$\begin{array}{l}\text { Waikar et al. } \\
\text { (61) }\end{array}$} & \multirow[t]{4}{*}{ ARF } & \multirow[t]{4}{*}{$\begin{array}{l}\text { Adults } \\
\text { hospitalized in } \\
\text { USA in } 2004\end{array}$} & \multirow[t]{4}{*}{99,629} & \multirow{4}{*}{$\begin{array}{l}\text { Discharge billing } \\
\text { codes, including ICD-9 } \\
\text { diagnostic and } \\
\text { procedure codes for } \\
\text { ARF }\end{array}$} & \multirow{4}{*}{$\begin{array}{l}\text { Linked laboratory and } \\
\text { hospital administrative } \\
\text { data with ARF defined } \\
\text { using nadir and peak } \\
\text { SCr: (1) } 100 \% \text { change } \\
\text { and (2) a variable } \\
\text { change (104, 105); } \\
\text { standardized review of } \\
300 \text { charts for ARF } \\
\text { requiring dialysis }\end{array}$} & & $\begin{array}{l}\text { Sensitivity-\% } \\
\text { (CI) }\end{array}$ & $\begin{array}{l}\text { Specificity-\% } \\
\text { (CI) }\end{array}$ & PPV-\% (Cl) & NPV-\% (Cl) & \multirow{4}{*}{$\begin{array}{l}\text { - Reference standard } \\
\text { based on laboratory } \\
\text { data may have resulted } \\
\text { in misclassification of } \\
\text { ARF as } \\
\text { diagnostic standard } \\
\text { - Sampling strategy for } \\
\text { chart review of ARF } \\
\text { requiring dialysis may } \\
\text { overestimate sensitivity }\end{array}$} \\
\hline & & & & & & $\mid$\begin{tabular}{|l} 
ARF $[$ by \\
Variable \\
Change \\
$(104,105)]$
\end{tabular} & 28.3 (CI NR) & 99.0 (CI NR) & 80.2 (Cl NR) & 91.0 (CI NR) & \\
\hline & & & & & & \begin{tabular}{|l|} 
ARF \\
(Requiring \\
Dialysis)
\end{tabular} & 90.4 (Cl NR) & 93.8 (Cl NR) & 94.0 (CI NR) & 90.0 (Cl NR) & \\
\hline & & & & & & \begin{tabular}{|l} 
ARF (by \\
$100 \%$ \\
Change)
\end{tabular} & 35.4 (Cl NR) & $97.7(\mathrm{Cl} N R)$ & 47.9 (Cl NR) & 96.1 (Cl NR) & \\
\hline \multirow[t]{3}{*}{$\begin{array}{l}\text { Blichert- } \\
\text { Hansen et al. } \\
(48)\end{array}$} & \multirow[t]{3}{*}{ AKI-RRT } & \multirow{3}{*}{$\begin{array}{l}\text { Adults } \\
\text { admitted to } \\
\text { ICU in } \\
\text { Denmark } \\
\text { between } 2005 \\
\text { and } 2010\end{array}$} & \multirow[t]{3}{*}{50} & \multirow{3}{*}{$\begin{array}{l}\text { Danish National Patient } \\
\text { Registry, which } \\
\text { includes discharge } \\
\text { procedure codes for } \\
\text { AKI-RRT }\end{array}$} & \multirow{3}{*}{$\begin{array}{l}\text { Standardized review of } \\
\text { medical charts for } \\
\text { ARF-RRT, including } \\
\text { acute HD or CRRT }\end{array}$} & & $\begin{array}{l}\text { Sensitivity-\% } \\
\text { (CI) }\end{array}$ & $\begin{array}{l}\text { Specificity-\% } \\
\text { (CI) }\end{array}$ & PPV-\% (Cl) & NPV-\% (Cl) & \multirow{3}{*}{$\begin{array}{l}\text { - Small sample size } \\
\text { limits generalizability } \\
\text { - Study limited to } \\
\text { AKI-RRT only } \\
\text { - Sensitivity, specificity, } \\
\text { and NPV could not } \\
\text { be reported }\end{array}$} \\
\hline & & & & & & AKI-RRT & NR & NR & $\begin{array}{l}98 \\
(91.0-99.8)\end{array}$ & NR & \\
\hline & & & & & & & & & & & \\
\hline \multirow[t]{3}{*}{$\begin{array}{l}\text { Schaffzin } \\
\text { et al. (49) }\end{array}$} & \multirow[t]{3}{*}{ AKI } & \multirow{3}{*}{\begin{tabular}{|l|} 
Children \\
hospitalized in \\
USA, exposed \\
to $3+$ \\
nephrotoxins \\
at once OR $3+$ \\
days of \\
aminoglycoside \\
therapy from \\
2011 to 2012
\end{tabular}} & \multirow{3}{*}{$\begin{array}{l}747 \text { from } \\
\text { retrospective } \\
\text { cohort; } 261 \text { from } \\
\text { prior prospective } \\
\text { cohort (106) } \\
\end{array}$} & \multirow{3}{*}{$\begin{array}{l}\text { Pediatric Health } \\
\text { Information System, } \\
\text { which includes } \\
\text { medication and } \\
\text { discharge billing codes } \\
\text { for AKI }\end{array}$} & \multirow{3}{*}{$\begin{array}{l}\text { Standardized review of } \\
\text { medical charts, merged } \\
\text { with electronic medical } \\
\text { record, using pRIFLE } \\
\text { definition (using SCr } \\
\text { criteria for R, I, and F } \\
\text { strata) }\end{array}$} & & $\begin{array}{l}\text { Sensitivity-\% } \\
\text { (CI) }\end{array}$ & $\begin{array}{l}\text { Specificity-\% } \\
\text { (CI) }\end{array}$ & PPV-\% (Cl) & NPV-\% (Cl) & \multirow{3}{*}{\begin{tabular}{|l} 
- Small sample size, \\
single-center data may \\
limit generalizability \\
- pRIFLE criteria may \\
still underestimate \\
nephrotoxin-associated \\
AKI without daily \\
screening, as in the \\
prospective cohort
\end{tabular}} \\
\hline & & & & & & $\begin{array}{l}\text { AKI (using } \\
\text { retrospective } \\
\text { cohort) }\end{array}$ & $e^{23.2(14.0-32.2)}$ & $95.0(93.1-96.6)$ & $\begin{array}{l}36.5 \\
(23.6-51.0)\end{array}$ & \begin{tabular}{|l}
90.9 \\
$(88.6-93.0)$
\end{tabular} & \\
\hline & & & & & & \begin{tabular}{|l|} 
AKI [using \\
prospective \\
cohort \\
$(106)]$
\end{tabular} & $21.4(11.8-31.0)$ & $94.2(90.1-97.5)$ & $\begin{array}{l}57.7 \\
(38.7-76.7)\end{array}$ & $\begin{array}{l}76.6 \\
(71.2-82.0)\end{array}$ & \\
\hline
\end{tabular}


TABLE 2 | Continued

\begin{tabular}{|c|c|c|c|c|c|c|c|c|c|c|c|}
\hline References & $\begin{array}{l}\text { Validated } \\
\text { Measure }\end{array}$ & $\begin{array}{l}\text { Study } \\
\text { Population }\end{array}$ & $\mathbf{N}$ & $\begin{array}{l}\text { Administrative } \\
\text { Health Data } \\
\text { Source }\end{array}$ & $\begin{array}{l}\text { Reference } \\
\text { Standard }\end{array}$ & & & Study Findings & & & Limitations \\
\hline \multirow[t]{4}{*}{$\begin{array}{l}\text { Tomlinson } \\
\text { et al. (50) }\end{array}$} & \multirow[t]{4}{*}{ AKI } & \multirow{4}{*}{$\begin{array}{l}\text { Adults without } \\
\text { ESRD } \\
\text { hospitalized in } \\
\text { England in } \\
2005 \text { and } \\
2010\end{array}$} & \multirow[t]{4}{*}{$\begin{array}{l}121 \text { (58 in 2005, } \\
63 \text { in 2010) }\end{array}$} & \multirow[t]{4}{*}{$\begin{array}{l}\text { Discharge billing codes, } \\
\text { including ICD-10 } \\
\text { diagnostic code for AKI }\end{array}$} & \multirow{4}{*}{$\begin{array}{l}\text { Standardized review of } \\
\text { selected medical charts } \\
\text { using KDIGO AKI } \\
\text { definition (using SCr } \\
\text { criteria) }\end{array}$} & & $\begin{array}{l}\text { Sensitivity-\% } \\
\text { (CI) }\end{array}$ & $\begin{array}{l}\text { Specificity-\% } \\
\text { (CI) }\end{array}$ & PPV-\% (Cl) & NPV-\% (CI) & \multirow{4}{*}{$\begin{array}{l}\text { - Small sample size, } \\
\text { single-center data may } \\
\text { limit generalizability } \\
\text { - One diagnostic code } \\
\text { examined only } \\
\text { - Sensitivity, specificity, } \\
\text { and NPV could not } \\
\text { be reported }\end{array}$} \\
\hline & & & & & & AKI & NR & NR & 95 (91-99) & NR & \\
\hline & & & & & & \begin{tabular}{|l} 
AKI, 2005 \\
Only
\end{tabular} & NR & NR & $95(89-100)$ & NR & \\
\hline & & & & & & \begin{tabular}{|l} 
AKI, 2010 \\
Only
\end{tabular} & NR & NR & $94(88-100)$ & NR & \\
\hline \multirow[t]{6}{*}{$\begin{array}{l}\text { Grams et al. } \\
\text { (51) }\end{array}$} & \multirow[t]{6}{*}{ AKI } & \multirow{6}{*}{$\begin{array}{l}\text { Adults without } \\
\text { ESRD } \\
\text { hospitalized in } \\
\text { the US } \\
\text { between } 1996 \\
\text { and 2008, } \\
\text { Atherosclerosis } \\
\text { Risk in } \\
\text { Communities } \\
\text { (ARIC) study } \\
\text { participants }\end{array}$} & \multirow[t]{6}{*}{10,056} & \multirow{6}{*}{$\begin{array}{l}\text { Discharge billing } \\
\text { codes, including ICD-9 } \\
\text { diagnostic and } \\
\text { procedure codes for } \\
\text { AKI }\end{array}$} & \multirow{6}{*}{$\begin{array}{l}\text { Standardized review of } \\
\text { selected medical charts } \\
\text { using KDIGO AKI } \\
\text { definition (using } \\
\text { SCr criteria) } \\
\text { Standardized review of } \\
\text { electronic medical } \\
\text { record using KDIGO } \\
\text { AKI definition (using } \\
\text { SCr or UO criteria) }\end{array}$} & & $\begin{array}{l}\text { Sensitivity-\% } \\
\text { (CI) }\end{array}$ & $\begin{array}{l}\text { Specificity-\% } \\
\text { (CI) }\end{array}$ & PPV-\% (Cl) & NPV-\% (CI) & \multirow{6}{*}{\begin{tabular}{|l} 
- Cohort admitted \\
between 1996 and \\
2008; nearly $50 \%$ of \\
hospitalizations used \\
for chart review were \\
between 1996 and \\
2002. Sensitivity \\
significantly increased \\
when comparing \\
hospitalizations \\
between 1996 and \\
2002 vs. 2002 and \\
$2008 ;$ age $<65$ vs. $\geq 65$ \\
years. This may reflect \\
increased \\
AKI awareness \\
- Electronic medical \\
record data is from one \\
center, which may \\
limit generalizability
\end{tabular}} \\
\hline & & & & & & \begin{tabular}{|l} 
AKI (vs. \\
Chart \\
Review)
\end{tabular} & $17.4(11.6-23.1)$ & ) 99.6 (99.3-99.9) & $\left|\begin{array}{l}92.0 \\
(85.9-98.2)\end{array}\right|$ & $\begin{array}{l}81.8 \\
(76.5-87.2)\end{array}$ & \\
\hline & & & & & & \begin{tabular}{|l} 
Stage 2 or \\
3 AKI (vs. \\
Chart \\
Review)
\end{tabular} & 40.3 (CI NR) & NR & NR & 99.9 (CI NR) & \\
\hline & & & & & & \begin{tabular}{|l|} 
Stage 3 AKI \\
(vs. Chart \\
Review) \\
\end{tabular} & 36.5 (CI NR) & 99.9 (Cl NR) & NR & NR & \\
\hline & & & & & & \begin{tabular}{|l} 
AKI (vs. \\
Electronic \\
Medical \\
Record) \\
\end{tabular} & $11.7(8.8-14.5)$ & $98.9(98.2-99.5)$ & \begin{tabular}{|l}
83.5 \\
$(75.4-91.7)$
\end{tabular} & $\begin{array}{l}69.3 \\
(66.9-71.7)\end{array}$ & \\
\hline & & & & & & & & & & & \\
\hline
\end{tabular}


TABLE 2 | Continued

\begin{tabular}{|c|c|c|c|c|c|c|c|c|c|c|c|}
\hline References & \begin{tabular}{|l|} 
Validated \\
Measure
\end{tabular} & \begin{tabular}{|l|} 
Study \\
Population
\end{tabular} & $\mathbf{N}$ & $\begin{array}{l}\text { Administrative } \\
\text { Health Data } \\
\text { Source }\end{array}$ & \begin{tabular}{|l|} 
Reference \\
Standard
\end{tabular} & & & Study Findings & & & Limitations \\
\hline \multirow[t]{3}{*}{$\begin{array}{l}\text { Maass et al. } \\
\text { (52) }\end{array}$} & \multirow[t]{3}{*}{ ARF } & \multirow{3}{*}{\begin{tabular}{|l|} 
Adults, aged \\
65 years or \\
older, \\
hospitalized in \\
Germany \\
between May- \\
December \\
2010
\end{tabular}} & \multirow[t]{3}{*}{$\begin{array}{l}3000 \text { (of which } \\
1500 \text { were } \\
\text { post-operative) }\end{array}$} & \multirow{3}{*}{$\begin{array}{l}\text { Discharge billing } \\
\text { codes, including } \\
\text { ICD-10 diagnostic and } \\
\text { procedure codes for } \\
\text { ARF }\end{array}$} & \multirow[t]{3}{*}{$\begin{array}{l}\text { Standardized review of } \\
\text { selected medical charts } \\
\text { for ARF }\end{array}$} & & $\begin{array}{l}\text { Sensitivity-\% } \\
\text { (CI) }\end{array}$ & $\begin{array}{l}\text { Specificity-\% } \\
\text { (CI) }\end{array}$ & PPV-\% (CI) & NPV-\% (Cl) & \multirow[t]{3}{*}{$\begin{array}{l}\text { - KDIGO criteria not } \\
\text { applied }\end{array}$} \\
\hline & & & & & & ARF & $17.6(12.7-24.1)$ & $99.2(98.7-99.4)$ & 56.6 (Cl NR) & NR & \\
\hline & & & & & & & & & & & \\
\hline \multirow[t]{7}{*}{$\begin{array}{l}\text { D'Arienzo } \\
\text { et al. (53) }\end{array}$} & \multirow[t]{7}{*}{ AKI } & \multirow{7}{*}{\begin{tabular}{|l|} 
Children (aged \\
0-18 years) \\
without ESRD \\
admitted to \\
pediatric ICU \\
in Canada \\
between 2003 \\
and 2005
\end{tabular}} & \multirow{7}{*}{$\begin{array}{l}2051 \text { ( } 355 \text { cardiac } \\
\text { surgery, } 1696 \\
\text { non-cardiac } \\
\text { surgery) }\end{array}$} & \multirow{7}{*}{$\begin{array}{l}\text { Provincial health care } \\
\text { administrative } \\
\text { database using ICD-9 } \\
\text { primary and secondary } \\
\text { diagnostic and dialysis } \\
\text { procedure codes }\end{array}$} & \multirow{7}{*}{$\begin{array}{l}\text { Standardized chart } \\
\text { review using KDIGO } \\
\text { AKI definition (using } \\
\text { SCr or UO criteria) and } \\
\text { severity }\end{array}$} & & $\begin{array}{l}\text { Sensitivity-\% } \\
\text { (Cl) }\end{array}$ & $\begin{array}{l}\text { Specificity-\% } \\
\text { (CI) }\end{array}$ & PPV-\% (CI) & NPV-\% (Cl) & \multirow{7}{*}{$\begin{array}{l}\text { - Small sample size for } \\
\text { cardiac surgery cohort } \\
\text { - Center-specific } \\
\text { practices may } \\
\text { limit generalizability } \\
\text { - Cohort admitted } \\
\text { between } 2003 \text { and } \\
\text { 2005; higher sensitivity } \\
\text { may be apparent } \\
\text { based on more current } \\
\text { practices and } \\
\text { increased } \\
\text { AKI awareness }\end{array}$} \\
\hline & & & & & & \begin{tabular}{|l} 
Cardiac \\
Surgery, \\
Any AKI
\end{tabular} & $5.9(4.7-7.6)$ & $100(99.2-100)$ & $\begin{array}{l}100 \\
(88.2-100)\end{array}$ & \begin{tabular}{|l}
49.4 \\
$(47.5-51.4)$
\end{tabular} & \\
\hline & & & & & & \begin{tabular}{|l} 
Cardiac \\
Surgery, \\
Stage 2 or \\
Worse AKI
\end{tabular} & $14.1(10.8-18.2)$ & $99.3(98.7-99.7)$ & \begin{tabular}{|l|}
81.8 \\
$(67.4-91.2)$
\end{tabular} & \begin{tabular}{|l}
84.0 \\
$(82.5-85.4)$
\end{tabular} & \\
\hline & & & & & & \begin{tabular}{|l} 
Cardiac \\
Surgery, \\
Stage 3 AKI
\end{tabular} & $14.3(9.7-20.5)$ & $98.1(97.3-98.7)$ & $\begin{array}{l}45.5 \\
(31.7-59.8)\end{array}$ & \begin{tabular}{|l|}
91.3 \\
$(90.0-92.4)$
\end{tabular} & \\
\hline & & & & & & \begin{tabular}{|l} 
Non- \\
Cardiac, \\
Any AKI
\end{tabular} & $13.8(12.6-15.1)$ & 99.1 (98.9-99.3) & $\begin{array}{l}82.4 \\
(78.2-85.8)\end{array}$ & \begin{tabular}{|l|}
78.5 \\
$(77.8-79.2)$
\end{tabular} & \\
\hline & & & & & & \begin{tabular}{|l|} 
Non- \\
Cardiac, \\
Stage 2 or \\
Worse AKI
\end{tabular} & $23.6(21.3-26.1)$ & $98.3(98.0-98.5)$ & $\begin{array}{l}61.8 \\
(57.0-66.3)\end{array}$ & $\begin{array}{l}91.7 \\
(91.1-92.1)\end{array}$ & \\
\hline & & & & & & \begin{tabular}{|l|} 
Non- \\
Cardiac, \\
Stage 3 AKI
\end{tabular} & $42.2(38.0-46.5)$ & ) $98.0(97.7-98.2)$ & $\begin{array}{l}51.5 \\
(46.7-56.2)\end{array}$ & \begin{tabular}{|l|}
97.1 \\
$(96.7-97.3)$
\end{tabular} & \\
\hline \begin{tabular}{|l} 
Etzioni et al. \\
$(54)$
\end{tabular} & ARF & \begin{tabular}{|l|} 
Adults \\
hospitalized for \\
surgical \\
operations \\
across 8 \\
hospitals in \\
USA between \\
2013 and \\
2015
\end{tabular} & $\begin{array}{l}41,432 \text { surgical } \\
\text { hospitalizations } \\
\text { (37\% general } \\
\text { surgery, } 20 \% \\
\text { orthopedic) }\end{array}$ & $\begin{array}{l}\text { Intra-hospital } \\
\text { administrative data } \\
\text { using ICD-9 codes } \\
\text { diagnostic and dialysis } \\
\text { procedure codes }\end{array}$ & $\begin{array}{l}\text { ACS' NSQIP } \\
\text { administrative data, } \\
\text { including patient } \\
\text { information, treatment, } \\
\text { and complications }\end{array}$ & \multicolumn{5}{|c|}{$\begin{array}{l}\text { Concordance (Cohen Kappa value) between administrative and ACS } \\
\text { NSQIP data for ARF: } 0.10 \text {. } \\
\text { Hospital-specific concordance was not statistically significant ( } P= \\
0.19 \text { ), suggestive of significant inter-hospital heterogeneity for ARF }\end{array}$} & $\begin{array}{l}\text { - Absence of a "gold } \\
\text { standard," Reference } \\
\text { standard did not use } \\
\text { chart reviewed data } \\
\text { and confirm AKI using } \\
\text { SCr or UO data } \\
\text { - Significant } \\
\text { inter-hospital variability } \\
\text { is likely contributory to } \\
\text { poor concordance }\end{array}$ \\
\hline
\end{tabular}




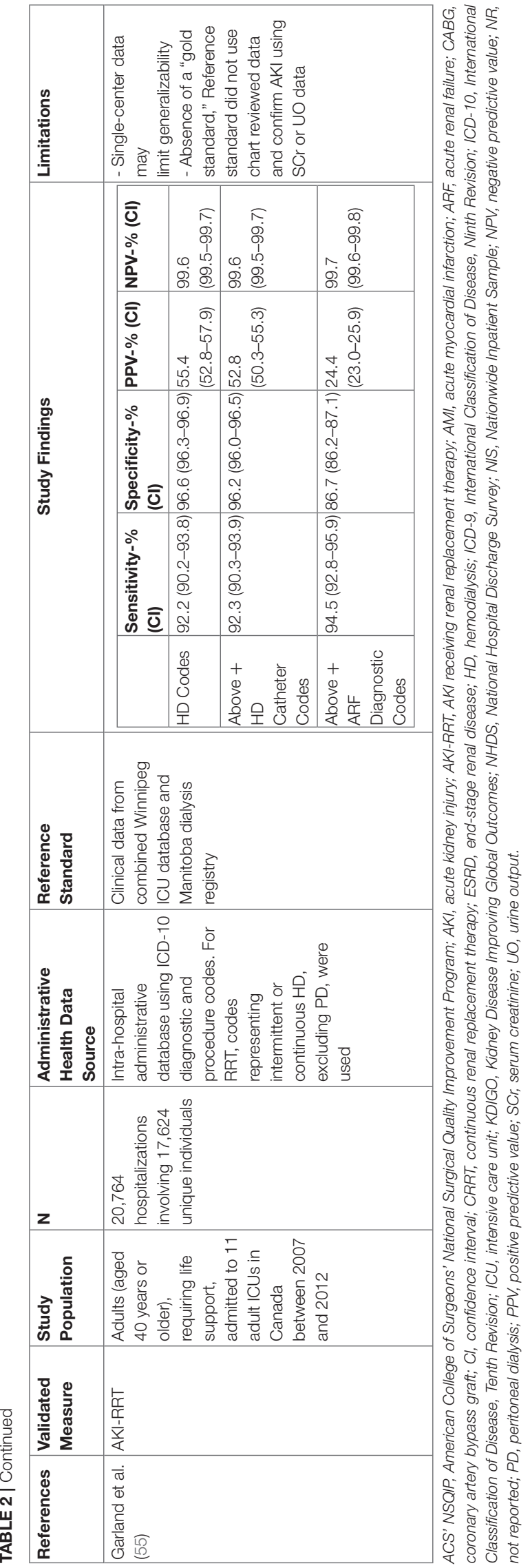

did not improve sensitivity of administrative health care data, relative to chart review, using creatinine only (sensitivity 11.7 vs. $17.4 \%)$ (51). Of note, the authors used KDIGO criteria for creatinine, but a more stringent definition for urine output, to improve sensitivity. In another study of children admitted to ICU, sensitivity was also not improved when using creatinine or urine output criteria, relative to creatinine alone (53). However, urine output criteria alone, relative to creatinine alone, had higher sensitivity for detecting any AKI (13.7 vs. 5.9\%) and for detecting stage 2 or worse AKI (18.2 vs. 14.1\%). NPV was more variable (49.4-99.7\%), but most reported an NPV of $>80 \%$. PPV was more variable (24.4-100\%), but most reported PPV of $>50 \%$. These results are consistent with those published in an earlier systematic review (47).

There are a number of limitations when validating administrative data for AKI. The most significant problems are related to the fact that the definition of AKI has changed significantly in the past 15 years, which is certainly a source of misclassification bias of AKI outcomes (16). Although oliguria has been associated with increased mortality in ICU populations and is an important component of the KDIGO definition $(3,63)$, urine output data are also difficult to extract from administrative databases and even chart review (51). Most validation studies have used creatinine criteria alone. Further validation studies are required to validate the use of billing codes using KDIGO criteria, different stages of severity, and inclusion of serum creatinine and/or urine output criteria for use in administrative health research.

There are additional challenges with validity when interpreting data based on physician billing codes. Physician billing practices change as our understanding of the significant morbidity and mortality associated with AKI evolves over time. For example, it is unclear whether practice changes are responsible for the increasing incidence of AKI observed from administrative health studies; however, the only study comparing administrative data at different time periods (2005 vs. 2010) did not show any significant change in PPV over time (50). Many administrative databases report only a limited number of billing codes; therefore, patients with milder AKI may be underrepresented. As well, billing codes have continued and will continue to change over time, requiring repeated validation of newer codes $(16,64)$. However, one study comparing administrative data derived from ICD-9 vs. ICD-10 billing codes showed no change in sensitivity $(80.0 \%)$ and only a small decrease in specificity ( 98.3 vs. $95.5 \%$ ), respectively (59).

Another major issue is limited generalizability, or external validity, given the significant heterogeneity of these studies (16). For example, the study by D'Arienzo et al. suggests that sensitivity may be lower in pediatric patients with milder AKI and/or postcardiac surgery (53). The study by Schaffzin et al. suggests that sensitivity is lower in pediatric patients with nephrotoxin-related AKI (49). These results reflect the significant heterogeneity in the populations studied, including age, disease severity, and etiology of AKI. Furthermore, practice standards are highly variable for the recognition and management of AKI, and this may be different across countries or academic vs. community centers. The reference standard is usually based on standardized 
chart review at one to two academic centers; these results may not be generalizable to nationwide practice for patients with AKI. Finally, administrative databases may not capture certain segments of the population in countries where there is both universal public and private health care systems and/or large uninsured populations (16).

In summary, these validation studies continue to be limited by variable definitions for AKI, significant heterogeneity of data sources and population studies, and reduced generalizability. Future studies are needed to further validate subpopulations, particularly in the pediatric population; as well, standardized guidelines for defining AKI using discharge billing codes will be essential in improving the applicability of administrative data to AKI health research (19).

\section{EPIDEMIOLOGY AND OUTCOMES OF AKI}

Administrative health research has served as an important methodological source for a number of observational epidemiology studies, which have fundamentally changed our understanding of AKI. This section will focus on studies in AKI conducted using administrative health data in the past 5 years.

\section{Incidence}

AKI is common in hospitalized patients with particularly high incidence rates seen in critically ill, post-cardiac surgery, oncology, and nephrotoxin-exposed adults and children $(1,3,62$, $65,66)$. However, there have been conflicting results regarding the changing temporal trends in AKI and AKI receiving KRT. One of the first studies used a national database to identify cases of AKI receiving KRT using ICD-9 codes and found that from 2000 to 2009, the incidence had increased by an average of $10 \%$ per year (67). Another study identified more than 18,000 adult patients with AKI receiving KRT using ICD-10 codes between 2000 and 2012 (68). The authors found that the crude incidence rate of AKI receiving KRT increased nearly 3-fold from 2000 to 2006; although the rate of growth remained stable between 2006 and 2012, the use of continuous renal replacement therapy (CRRT) increased throughout this period and especially in patients $>75$ years with high comorbid disease. In children, a similar trend of dialysis receiving AKI has been reported (69). Our group found an increasing trend in the incidence of AKI, as well as use of hemodialysis and CRRT among hospitalized children (1 month to 18 years) in Ontario, Canada (69). However, another large study, which compared the annual AKI incidence rate using data from an electronic health record surveillance tool and administrative data (70), found no difference in AKI incidence between 2006 and 2014 when adjusted for age and sex using either data source.

\section{Short-Term Outcomes}

Health administrative databases have improved our understanding that $\mathrm{AKI}$ is independently associated with increased hospital mortality and morbidity, including length of hospital and ICU stay, and increased health care costs. One study in Japan across more than 280 hospitals showed a decreasing trend in crude in-hospital mortality from $45 \%$ in 2007 to $36 \%$ in 2016 (71). An Italian study showed in-hospital mortality rate of nearly $30 \%$ with the highest risk being in patients with AKI receiving KRT [odds ratio, 2.7; 95\% confidence interval (CI), 2.7-2.8] (72). Finally, another Canadian study showed mortality rates between 30 and $40 \%$ for adult patients with AKI receiving KRT, but no association with increased mortality in centers who manage a lower volume of patients requiring KRT (73). The Canadian study in children with AKI receiving KRT also examined 30-day mortality, finding an increased rate from 14 to $25 \%$ between 1996 and 2009 , although this rate subsequently decreased to $19 \%$ by 2015 (69). Another study in Japan included pediatric patients ( $>12$ years) and reported $50 \%$ in-hospital mortality rate for patients treated with CRRT (74).

Several studies have also described rates of renal recovery, as well as the recurrence risk after AKI, both of which reflect a growing appreciation for AKI as a dynamic process (7578). A study of critically ill children in Canada showed that children with stage 3 AKI were more than 3-fold likely to have elevated serum creatinine at discharge $(>1.5 \times$ baseline $)$, relative to those without stage 3 AKI (79). A small study examined children who received ventricular assist devices (VADs) for heart failure and subsequently went on to undergo heart transplant (80). Those children without renal recovery (serum creatinine $\geq 1.5 \times$ baseline) 7 days after VAD implantation, relative to children who had full recovery, were not at increased risk for $\mathrm{CKD}$ at 1 year following heart transplant; however, those with reduced estimated glomerular filtration rate 1 month after VAD implantation were at increased risk for CKD.

There is also increased interest in the relative cost and burden associated with AKI. One study in Alberta, Canada, estimated the incremental cost of AKI to be more than Canadian $\$ 200$ million per year (81). Patients with AKI receiving KRT had prolonged length of stay by more than 7 days and increased cost by up to $\$ 20,000$, relative to patients without AKI. Another large study of adults from Alberta, Canada, undergoing cardiac surgery also showed increased length of stay and costs associated with increased AKI severity (82).

\section{Risk Factors}

Risk factors for AKI in hospitalized patients include extremes of age, underlying illness (i.e., sepsis, cardiovascular disease with or without bypass surgery, oncologic disease), nephrotoxin exposure, inflammatory mediators (i.e., cytokine release), and disease severity $(62,75,83-89)$. A large study in the United States of 3.6 million postsurgical patients found that AKI was common postoperatively, affecting more than $10 \%$ of hospitalized patients (86). AKI in patients with CKD is also an important risk factor for subsequent progression to ESKD (88). One study highlighted that patients who progressed from stage 3 to stage 4 CKD also had a high risk for AKI (89). Other subpopulations noted to be at increased risk for AKI-related morbidity and mortality include adults with decompensated liver disease (90) and stroke (91).

A number of studies have examined the impact of nephrotoxic medications using administrative health data (92-98). One study in hospitalized children across six of the largest children's hospitals in the United States found that combined use of vancomycin with piperacillin/tazobactam conferred more than 3-fold increased risk for antibiotic-associated AKI, relative 
to vancomycin with another antipseudomonal antibiotic (93). Other nephrotoxins examined include non-steroidal antiinflammatory drugs in young, healthy adults (94), brands and dosing of immunoglobulin products (95), and statin use in elderly patients (96), all of which showed modest associations with AKI. Administrative data were also used to develop an evidence-based nephrotoxin medication list that could be used as part of a screening program for AKI in children (98).

\section{Long-Term Outcomes}

AKI is associated with increased risk for development of cardiovascular disease, CKD, and ESKD in adults $(1,75,78,83$, 99). A study in Northern California, including more than 43,000 patients, showed that AKI in adults is independently associated with HTN as early as 6 months after initial event (99). Another large study of more than 100,000 patients in the United States showed that more than $30 \%$ of hospitalized adults with AKI went on to develop CKD at 1-year follow-up (78).

The risks of long-term kidney and cardiovascular sequelae after pediatric AKI remain uncertain, at least partially due to the fact that studies have lacked comparator cohorts, have had high losses to follow-up, and/or have had short followup periods. Recent prospective cohort studies have conflicting results; TRIBE-AKI, FRAIL-AKI, and ASSESS-AKI all found that cardiac surgery-associated AKI survivors have similar 4-7-year kidney outcomes vs. children without AKI (24-26), whereas Benisty et al. found higher long-term risks of CKD and HTN among survivors of ICU-associated AKI (27). Although there is a clear signal of increased CKD risk among pediatric AKI survivors in other cohort studies $(6,13,28-30)$, the long-term outcomes after episodes of dialysis-receiving AKI remain uncertain.

Health administrative databases provide a unique opportunity to follow children many years after an episode of AKI, even after the age of 18 years, to study their long-term outcomes. Moreover, CKD and HTN-related cardiovascular changes start early in life; therefore, it is essential to understand the timing and magnitude of the onset of these events after an episode of AKI, so that appropriate treatment can be initiated in time to address these risk factors and avoid or delay future cardiovascular disease (100). Current AKI guidelines in neonates and children do not provide recommendations for long-term follow-up, primarily due to a lack of studies with robust data.

Several studies have examined long-term outcomes in hospitalized children with AKI. A recent study from Ontario using administrative health data with median 10-year follow-up compared children surviving AKI receiving KRT with hospitalmatched controls (101). The authors found that these children had significantly increased hazard for major adverse kidney events (composite outcome of kidney failure and all-cause mortality) [adjusted hazard ratio (HR), 4.97; 95\% CI, 4.046.10], CKD (adjusted HR, 8.70; 95\% CI, 6.68-11.34), and HTN (adjusted HR, 3.35; 95\% CI, 2.59-4.33). Another cohort of critically ill children with AKI in Quebec was also found to have increased risk for mortality 5-7 years following hospital discharge, relative to children without AKI (adjusted HR, 3.1; 95\% CI, 1.5-6.6) (6). Health care utilization was also increased for critically ill children with AKI, relative to children without AKI, including increased hospitalizations and physician visits at 5 years following hospital discharge (7). This cohort also had increased risk for HTN (adjusted HR, 2.19; 95\% CI, 1.47-3.26) (9) and CKD (adjusted HR, 2.2; 95\% CI, 1.1-4.5) (8) at 5-year follow-up, defined using administrative health care data. Another study following critically ill children with congenital heart disease showed that those who developed AKI within 5 days of cardiac surgery were at significantly increased risk for CKD at 5 years, relative to those who did not develop AKI ( $\mathrm{HR}, 3.8 ; 95 \% \mathrm{CI}$, 1.4-10.4) (102). We have also previously reported that, among children who underwent cardiac surgery for congenital heart disease, those who received dialysis for AKI during their index cardiac surgery admission were at a 5-fold higher risk of ESKD (crude HR, 5.0; 95\% CI, 2.0-12.6) compared with those who did not receive dialysis during their cardiac surgery admission (103).

\section{CONCLUSION}

Our understanding of AKI has undergone a significant transformation over the past 15 years partly due to methodological advances in which AKI has been studied by epidemiologists (1). Hand-in-hand with a veritable explosion of research demonstrating the morbidity and mortality related to AKI in adults and children, there has been significant attention paid to "big data" $(18,25,37)$. Registries containing administrative health care data are readily available, efficient, and large $(14,19)$. Validation studies have been shown that administrative health care data are highly specific, despite having modest sensitivity, for the diagnosis of AKI. There are important limitations as well, including heterogeneity of data sources and inconsistent reporting on specific outcome measures (i.e., quality of life, patient-reported outcomes). The statements from the ADQI consensus conference recognized many strategies by which administrative health data could be applied to AKI-specific research studies and address knowledge gaps in the field (18-23).

Studies using administrative health care data have expanded our understanding of the incidence, risk factors, and outcomes of AKI in both adults and children. Importantly, we have demonstrated that these clinically relevant findings have been supported by results from traditional data sources. Continued progress in the application of large data sources to epidemiological studies will continue to further our understanding of important patient-related outcomes of adults and children with AKI.

\section{AUTHOR CONTRIBUTIONS}

EU drafted the manuscript, prepared the figures, and reviewed and revised the manuscript. GS also contributed to the manuscript and reviewed and revised the manuscript. RC supervised the manuscript preparation and reviewed and revised the manuscript for intellectual content. MZ reviewed and revised the manuscript for intellectual content. All authors approved the final manuscript as submitted. 


\section{REFERENCES}

1. Chawla LS, Bellomo R, Bihorac A, Goldstein SL, Siew ED, Bagshaw SM, et al. Acute kidney disease and renal recovery: consensus report of the Acute Disease Quality Initiative (ADQI) 16 Workgroup. Nat Rev Nephrol. (2017) 13:241-57. doi: 10.1038/nrneph.2017.2

2. Zappitelli M, Moffett BS, Hyder A, Goldstein SL. Acute kidney injury in non-critically ill children treated with aminoglycoside antibiotics in a tertiary healthcare centre: a retrospective cohort study. Nephrol Dial Transplant. (2011) 26:144-50. doi: 10.1093/ndt/gfq375

3. Kaddourah A, Basu RK, Bagshaw SM, Goldstein SL. Epidemiology of acute kidney injury in critically Ill children and young adults. N Engl J Med. (2017) 376:11-20. doi: 10.1056/NEJMoa1611391

4. Li S, Krawczeski CD, Zappitelli M, Devarajan P, Thiessen-Philbrook H, Coca SG, et al. Incidence, risk factors, and outcomes of acute kidney injury after pediatric cardiac surgery: a prospective multicenter study. Crit Care Med. (2011) 39:1493-9. doi: 10.1097/CCM.0b013e31821201d3

5. Coca SG, Singanamala S, Parikh CR. Chronic kidney disease after acute kidney injury: a systematic review and meta-analysis. Kidney Int. (2012) 81:442-8. doi: 10.1038/ki.2011.379

6. Hessey E, Morissette G, Lacroix J, Perreault S, Samuel S, Dorais M, et al. Long-term mortality after acute kidney injury in the pediatric ICU. Hosp Pediatr. (2018) 8:260-8. doi: 10.1542/hpeds.2017-0215

7. Hessey E, Morissette G, Lacroix J, Perreault S, Samuel S, Dorais M, et al. Healthcare utilization after acute kidney injury in the pediatric intensive care unit. Clin J Am Soc Nephrol. (2018) 13:685-92. doi: 10.2215/CJN.09350817

8. Hessey E, Perreault S, Dorais M, Roy L, Zappitelli M. Acute kidney injury in critically Ill children and subsequent chronic kidney disease. Can J Kidney Health Dis. (2019) 6:2054358119880188. doi: 10.1177/2054358119880188

9. Hessey E, Perreault S, Roy L, Dorais M, Samuel S, Phan V, et al. Acute kidney injury in critically ill children and 5-year hypertension. Pediatr Nephrol. (2020) 35:1097-107. doi: 10.1007/s00467-020-04488-5

10. Misra PS, Silva ESV, Collister D. Roadblocks and opportunities to the implementation of novel therapies for acute kidney injury: a narrative review. Can J Kidney Health Dis. (2019) 6:2054358119880519. doi: 10.1177/2054358119880519

11. Mazzali C, Duca P. Use of administrative data in healthcare research. Intern Emerg Med. (2015) 10:517-24. doi: 10.1007/s11739-015-1213-9

12. Cadarette SM, Wong L. An introduction to health care administrative data. Can J Hosp Pharm. (2015) 68:232-7. doi: 10.4212/cjhp.v68i3.1457

13. Lucyk K, Tang K, Quan H. Barriers to data quality resulting from the process of coding health information to administrative data: a qualitative study. $B M C$ Health Serv Res. (2017) 17:766. doi: 10.1186/s12913-017-2697-y

14. Sutherland SM. Big data and pediatric acute kidney injury: the promise of electronic health record systems. Front Pediatr. (2019) 7:536. doi: 10.3389/fped.2019.00536

15. Skull SA, Andrews RM, Byrnes GB, Campbell DA, Nolan TM, Brown $\mathrm{GV}$, et al. ICD-10 codes are a valid tool for identification of pneumonia in hospitalized patients aged $>$ or $=65$ years. Epidemiol Infect. (2008) 136:232-40. doi: 10.1017/S0950268807008564

16. Gavrielov-Yusim N, Friger M. Use of administrative medical databases in population-based research. J Epidemiol Community Health. (2014) 68:2837. doi: 10.1136/jech-2013-202744

17. O'Malley KJ, Cook KF, Price MD, Wildes KR, Hurdle JF, Ashton CM. Measuring diagnoses: ICD code accuracy. Health Serv Res. (2005) 40:162039. doi: 10.1111/j.1475-6773.2005.00444.x

18. Bagshaw SM, Goldstein SL, Ronco C, Kellum JA, Group AC. Acute kidney injury in the era of big data: the 15th Consensus Conference of the Acute Dialysis Quality Initiative (ADQI). Can J Kidney Health Dis. (2016) 3:5. doi: 10.1186/s40697-016-0103-z

19. Siew ED, Basu RK, Wunsch H, Shaw AD, Goldstein SL, Ronco C, et al. Optimizing administrative datasets to examine acute kidney injury in the era of big data: workgroup statement from the 15th ADQI Consensus Conference. Can J Kidney Health Dis. (2016) 3:12. doi: 10.1186/s40697-016-0098-5

20. James MT, Hobson CE, Darmon M, Mohan S, Hudson D, Goldstein SL, et al. Applications for detection of acute kidney injury using electronic medical records and clinical information systems: workgroup statements from the 15th ADQI Consensus Conference. Can J Kidney Health Dis. (2016) 3:9. doi: 10.1186/s40697-016-0100-2

21. Hoste EA, Kashani K, Gibney N, Wilson FP, Ronco C, Goldstein SL, et al. Impact of electronic-alerting of acute kidney injury: workgroup statements from the 15th ADQI Consensus Conference. Can J Kidney Health Dis. (2016) 3:10. doi: 10.1186/s40697-016-0101-1

22. Sutherland SM, Chawla LS, Kane-Gill SL, Hsu RK, Kramer AA, Goldstein SL, et al. Utilizing electronic health records to predict acute kidney injury risk and outcomes: workgroup statements from the 15th ADQI Consensus Conference. Can J Kidney Health Dis. (2016) 3:11. doi: 10.1186/s40697-016-0099-4

23. Mehta R, Bihorac A, Selby NM, Quan H, Goldstein SL, Kellum JA, et al. Establishing a continuum of acute kidney injury - tracing AKI using data source linkage and long-term follow-up: Workgroup Statements from the 15th ADQI Consensus Conference. Can J Kidney Health Dis. (2016) 3:13. doi: 10.1186/s40697-016-0102-0

24. Nguyen LL, Barshes NR. Analysis of large databases in vascular surgery. $J$ Vasc Surg. (2010) 52:768-74. doi: 10.1016/j.jvs.2010.03.027

25. Ehrenstein V, Nielsen H, Pedersen AB, Johnsen SP, Pedersen L. Clinical epidemiology in the era of big data: new opportunities, familiar challenges. Clin Epidemiol. (2017) 9:245-50. doi: 10.2147/CLEP.S129779

26. Al-Jaishi AA, McIntyre CW, Sontrop JM, Dixon SN, Anderson S, Bagga $A$, et al. major outcomes with personalized dialysate TEMPerature (MyTEMP): rationale and design of a pragmatic, registry-based, cluster randomized controlled trial. Can J Kidney Health Dis. (2020) 7:2054358119887988. doi: 10.1177/2054358119887988

27. Ishiguro L, Saskin R, Vermeulen MJ, Yates E, Gunraj N, Victor JC. Increasing access to health administrative data with ICES data \& analytic services. Healthc Q. (2016) 19:7-9. doi: 10.12927/hcq.2016.24617

28. Chiu ATG, Chung CCY, Wong WHS, Lee SL, Chung BHY. Healthcare burden of rare diseases in Hong Kong - adopting ORPHAcodes in ICD10 based healthcare administrative datasets. Orphanet J Rare Dis. (2018) 13:147. doi: 10.1186/s13023-018-0892-5

29. Chibnik LB, Massarotti EM, Costenbader KH. Identification and validation of lupus nephritis cases using administrative data. Lupus. (2010) 19:7413. doi: $10.1177 / 0961203309356289$

30. Evans E, Grella CE, Murphy DA, Hser YI. Using administrative data for longitudinal substance abuse research. J Behav Health Serv Res. (2010) 37:252-71. doi: 10.1007/s11414-008-9125-3

31. Pannu N, James M, Hemmelgarn B, Klarenbach S, Network AKD. Association between AKI, recovery of renal function, and long-term outcomes after hospital discharge. Clin J Am Soc Nephrol. (2013) 8:194202. doi: $10.2215 / \mathrm{CJN} .06480612$

32. Edelman LS, Guo JW, Fraser A, Beck SL. Linking clinical research data to population databases. Nurs Res. (2013) 62:438-44. doi: 10.1097/NNR.0000000000000002

33. Roos LL, Nicol JP, Cageorge SM. Using administrative data for longitudinal research: comparisons with primary data collection. J Chronic Dis. (1987) 40:41-9. doi: 10.1016/0021-9681(87)90095-6

34. Rezai MR, Maclagan LC, Donovan LR, Tu JV. Classification of Canadian immigrants into visible minority groups using country of birth and mother tongue. Open Med. (2013) 7:e85-93.

35. Rosella LC, Fitzpatrick T, Wodchis WP, Calzavara A, Manson H, Goel V. High-cost health care users in Ontario, Canada: demographic, socioeconomic, and health status characteristics. BMC Health Serv Res. (2014) 14:532. doi: 10.1186/s12913-014-0532-2

36. Tu K, Mitiku TF, Ivers NM, Guo H, Lu H, Jaakkimainen L, et al. Evaluation of Electronic Medical Record Administrative data Linked Database (EMRALD). Am J Manag Care. (2014) 20:e15-21.

37. Sutherland SM, Goldstein SL, Bagshaw SM. Leveraging big data and electronic health records to enhance novel approaches to acute kidney injury research and care. Blood Purif. (2017) 44:68-76. doi: 10.1159/000458751

38. Motheral BR, Fairman KA. The use of claims databases for outcomes research: rationale, challenges, and strategies. Clin Ther. (1997) 19:34666. doi: 10.1016/S0149-2918(97)80122-1

39. Mazzali C, Paganoni AM, Ieva F, Masella C, Maistrello M, Agostoni $\mathrm{O}$, et al. Methodological issues on the use of administrative data in healthcare research: the case of heart failure hospitalizations 
in Lombardy region, 2000 to 2012. BMC Health Serv Res. (2016) 16:234. doi: 10.1186/s12913-016-1489-0

40. Sharabiani MT, Aylin P, Bottle A. Systematic review of comorbidity indices for administrative data. Med Care. (2012) 50:1109-18. doi: 10.1097/MLR.0b013e31825f64d0

41. Peabody JW, Luck J, Jain S, Bertenthal D, Glassman P. Assessing the accuracy of administrative data in health information systems. Med Care. (2004) 42:1066-72. doi: 10.1097/00005650-200411000-00005

42. Fung KW, Richesson R, Smerek M, Pereira KC, Green BB, Patkar A, et al. Preparing for the ICD-10-CM transition: automated methods for translating ICD codes in clinical phenotype definitions. EGEMS. (2016) 4:1211. doi: 10.13063/2327-9214.1211

43. Cella D, Riley W, Stone A, Rothrock N, Reeve B, Yount S, et al. The Patient-Reported Outcomes Measurement Information System (PROMIS) developed and tested its first wave of adult self-reported health outcome item banks: 2005-2008. J Clin Epidemiol. (2010) 63:1179-94. doi: 10.1016/j.jclinepi.2010.04.011

44. Shiff NJ, Jama S, Boden C, Lix LM. Validation of administrative health data for the pediatric population: a scoping review. BMC Health Serv Res. (2014) 14:236. doi: 10.1186/1472-6963-14-236

45. Brookhart MA, Stürmer T, Glynn RJ, Rassen J, Schneeweiss S. Confounding control in healthcare database research: challenges and potential approaches. Med Care. (2010) 48:S114-20. doi: 10.1097/MLR.0b013e3181dbebe3

46. Geraci JM, Ashton CM, Kuykendall DH, Johnson ML, Wu L. International Classification of Diseases, 9th Revision, Clinical Modification codes in discharge abstracts are poor measures of complication occurrence in medical inpatients. Med Care. (1997) 35:589-602. doi: 10.1097/00005650-199706000-00005

47. Vlasschaert ME, Bejaimal SA, Hackam DG, Quinn R, Cuerden MS, Oliver MJ, et al. Validity of administrative database coding for kidney disease: a systematic review. Am J Kidney Dis. (2011) 57:29-43. doi: 10.1053/j.ajkd.2010.08.031

48. Blichert-Hansen L, Nielsson MS, Nielsen RB, Christiansen CF, Norgaard M. Validity of the coding for intensive care admission, mechanical ventilation, and acute dialysis in the Danish National Patient Registry: a short report. Clin Epidemiol. (2013) 5:9-12. doi: 10.2147/CLEP.S37763

49. Schaffzin JK, Dodd CN, Nguyen H, Schondelmeyer A, Campanella S, Goldstein SL. Administrative data misclassifies and fails to identify nephrotoxin-associated acute kidney injury in hospitalized children. Hosp Pediatr. (2014) 4:159-66. doi: 10.1542/hpeds.2013-0116

50. Tomlinson LA, Riding AM, Payne RA, Abel GA, Tomson CR, Wilkinson IB, et al. The accuracy of diagnostic coding for acute kidney injury in England - a single centre study. BMC Nephrol. (2013) 14:58. doi: 10.1186/1471-2369-14-58

51. Grams ME, Waikar SS, MacMahon B, Whelton S, Ballew SH, Coresh J. Performance and limitations of administrative data in the identification of AKI. Clin J Am Soc Nephrol. (2014) 9:682-9. doi: 10.2215/CJN.07650713

52. Maass C, Kuske S, Lessing C, Schrappe M. Are administrative data valid when measuring patient safety in hospitals? A comparison of data collection methods using a chart review and administrative data. Int J Qual Health Care. (2015) 27:305-13. doi: 10.1093/intqhe/ mzv045

53. D’Arienzo D, Hessey E, Ali R, Perreault S, Samuel S, Roy L, et al. A validation study of administrative health care data to detect acute kidney injury in the pediatric intensive care unit. Can J Kidney Health Dis. (2019) 6:2054358119827525. doi: 10.1177/2054358119827525

54. Etzioni DA, Lessow C, Bordeianou LG, Kunitake H, Deery SE, Carchman E, et al. Concordance Between Registry and Administrative Data in the Determination of Comorbidity: a Multi-institutional Study. Ann Surg. (2019) 272:1006-11. doi: 10.1097/SLA.0000000000003247

55. Garland A, Marrie RA, Wunsch H, Yogendran M, Chateau D. Accuracy of administrative hospital data to identify use of life support modalities. A Canadian study. Ann Am Thorac Soc. (2020) 17:229-35. doi: 10.1513/AnnalsATS.201902-106OC

56. Quan H, Parsons GA, Ghali WA. Assessing accuracy of diagnosis-type indicators for flagging complications in administrative data. J Clin Epidemiol. (2004) 57:366-72. doi: 10.1016/j.jclinepi.2003.01.002
57. Liangos O, Wald R, O’Bell JW, Price L, Pereira BJ, Jaber BL. Epidemiology and outcomes of acute renal failure in hospitalized patients: a national survey. Clin J Am Soc Nephrol. (2006) 1:43-51. doi: 10.2215/CJN.00220605

58. Parker JP, Li Z, Damberg CL, Danielsen B, Carlisle DM. Administrative versus clinical data for coronary artery bypass graft surgery report cards: the view from California. Med Care. (2006) 44:687-95. doi: 10.1097/01.mlr.0000215815.70506.b6

59. So L, Evans D, Quan H. ICD-10 coding algorithms for defining comorbidities of acute myocardial infarction. BMC Health Serv Res. (2006) 6:161. doi: 10.1186/1472-6963-6-161

60. Waikar SS, Curhan GC, Wald R, McCarthy EP, Chertow GM. Declining mortality in patients with acute renal failure, 1988 to 2002. J Am Soc Nephrol. (2006) 17:1143-50. doi: 10.1681/ASN.2005091017

61. Waikar SS, Wald R, Chertow GM, Curhan GC, Winkelmayer WC, Liangos $\mathrm{O}$, et al. Validity of international classification of diseases, ninth revision, clinical modification codes for acute renal failure. J Am Soc Nephrol. (2006) 17:1688-94. doi: 10.1681/ASN.2006010073

62. Khwaja A. KDIGO clinical practice guidelines for acute kidney injury. Nephron Clin Pract. (2012) 120:c179-84. doi: 10.1159/000339789

63. Macedo E, Malhotra R, Bouchard J, Wynn SK, Mehta RL. Oliguria is an early predictor of higher mortality in critically ill patients. Kidney Int. (2011) 80:760-7. doi: 10.1038/ki.2011.150

64. Ambroggio LV, Shah SS. Administrative data: expanding the infrastructure for pediatric research. J Pediatr. (2013) 162:6814. doi: 10.1016/j.jpeds.2012.10.040

65. Mehta RL, Cerdá J, Burdmann EA, Tonelli M, García-García G, Jha V, et al. International Society of Nephrology's 0by 25 initiative for acute kidney injury (zero preventable deaths by 2025): a human rights case for nephrology. Lancet. (2015) 385:2616-43. doi: 10.1016/S0140-6736(15)60126-X

66. Jetton JG, Boohaker LJ, Sethi SK, Wazir S, Rohatgi S, Soranno DE, et al. Incidence and outcomes of neonatal acute kidney injury (AWAKEN): a multicentre, multinational, observational cohort study. Lancet Child Adolesc Health. (2017) 1:184-94. doi: 10.1016/S2352-4642(17)30069-X

67. Hsu RK, McCulloch CE, Dudley RA, Lo LJ, Hsu CY. Temporal changes in incidence of dialysis-requiring AKI. J Am Soc Nephrol. (2013) 24:3742. doi: 10.1681/ASN.2012080800

68. Carlson N, Hommel K, Olesen JB, Soja AM, Vilsboll T, Kamper AL, et al. Dialysis-Requiring acute kidney injury in Denmark 2000-2012: time trends of incidence and prevalence of risk factors-a nationwide study. PLOS ONE. (2016) 11:e0148809. doi: 10.1371/journal.pone.0148809

69. Chanchlani R, Nash DM, McArthur E, Zappitelli M, Archer V, Kuwornu $J P$, et al. Secular trends in incidence, modality and mortality with dialysis receiving aki in children in Ontario: a population-based cohort study. Clin J Am Soc Nephrol. (2019) 14:1288-96. doi: 10.2215/CJN.08 250718

70. Kashani K, Shao M, Li G, Williams AW, Rule AD, Kremers WK, et al. No increase in the incidence of acute kidney injury in a population-based annual temporal trends epidemiology study. Kidney Int. (2017) 92:7218. doi: 10.1016/j.kint.2017.03.020

71. Miyamoto $Y$, Iwagami M, Aso S, Yasunaga $H$, Matsui $H$, Fushimi $K$, et al. Temporal change in characteristics and outcomes of acute kidney injury on renal replacement therapy in intensive care units: analysis of a nationwide administrative database in Japan, 2007-2016. Crit Care. (2019) 23:172. doi: 10.1186/s13054-019-2468-8

72. Fabbian F, Savrie C, De Giorgi A, Cappadona R, Di Simone E, Boari B, et al. Acute kidney injury and in-hospital mortality: a retrospective analysis of a nationwide administrative database of elderly subjects in Italy. J Clin Med. (2019) 8:1371. doi: $10.3390 / \mathrm{jcm} 8091371$

73. Chimunda T, Silver SA, Kuwornu JP, Li L, Nash DM, Dixon SN, et al. Hospital case volume and clinical outcomes in critically ill patients with acute kidney injury treated with dialysis. J Crit Care. (2018) 48:27682. doi: $10.1016 /$ j.jcrc.2018.08.024

74. Iwagami M, Yasunaga H, Noiri E, Horiguchi H, Fushimi K, Matsubara $\mathrm{T}$, et al. Current state of continuous renal replacement therapy for acute kidney injury in Japanese intensive care units in 2011: analysis of a national administrative database. Nephrol Dial Transplant. (2015) 30:98895. doi: $10.1093 / \mathrm{ndt} / \mathrm{gfv} 069$ 
75. Forni LG, Darmon M, Ostermann M, Oudemans-van Straaten HM, Pettila $\mathrm{V}$, Prowle JR, et al. Renal recovery after acute kidney injury. Intensive Care Med. (2017) 43:855-66. doi: 10.1007/s00134-017-4809-x

76. Kellum JA, Sileanu FE, Bihorac A, Hoste EAJ, Chawla LS. Recovery after acute kidney injury. Am J Respir Crit Care Med. (2017) 195:78491. doi: 10.1164/rccm.201604-0799OC

77. Korenkevych D, Ozrazgat-Baslanti T, Thottakkara P, Hobson CE, Pardalos P, Momcilovic $\mathrm{P}$, et al. The pattern of longitudinal change in serum creatinine and 90-day mortality after major surgery. Ann Surg. (2016) 263:121927. doi: 10.1097/SLA.0000000000001362

78. Heung M, Steffick DE, Zivin K, Gillespie BW, Banerjee T, Hsu CY, et al. Acute kidney injury recovery pattern and subsequent risk of CKD: an analysis of veterans health administration data. Am J Kidney Dis. (2016) 67:742-52. doi: 10.1053/j.ajkd.2015.10.019

79. Hessey E, Ali R, Dorais M, Morissette G, Pizzi M, Rink N, et al. Renal function follow-up and renal recovery after acute kidney injury in critically Ill children. Pediatr Crit Care Med. (2017) 18:733-40. doi: 10.1097/PCC.0000000000001166

80. Hollander SA, Cantor RS, Sutherland SM, Koehl DA, Pruitt E, McDonald $\mathrm{N}$, et al. Renal injury and recovery in pediatric patients after ventricular assist device implantation and cardiac transplant. Pediatr Transplant. (2019) 23:e13477. doi: 10.1111/petr.13477

81. Collister D, Pannu N, Ye F, James M, Hemmelgarn B, Chui B, et al. Health care costs associated with AKI. Clin J Am Soc Nephrol. (2017) 12:173343. doi: 10.2215/CJN.00950117

82. Lau D, Pannu N, James MT, Hemmelgarn BR, Kieser TM, Meyer SR, et al. Costs and consequences of acute kidney injury after cardiac surgery: a cohort study. J Thorac Cardiovasc Surg. (2020) 162:8807. doi: 10.1016/j.jtcvs.2020.01.101

83. Chawla LS, Eggers PW, Star RA, Kimmel PL. Acute kidney injury and chronic kidney disease as interconnected syndromes. N Engl J Med. (2014) 371:58-66. doi: 10.1056/NEJMra1214243

84. Hoste EAJ, Kellum JA, Selby NM, Zarbock A, Palevsky PM, Bagshaw SM, et al. Global epidemiology and outcomes of acute kidney injury. Nat Rev Nephrol. (2018) 14:607-25. doi: 10.1038/s41581-018-0052-0

85. Silver SA, Harel Z, McArthur E, Nash DM, Acedillo R, Kitchlu A, et al. Causes of death after a hospitalization with AKI. J Am Soc Nephrol. (2018) 29:1001-10. doi: 10.1681/ASN.2017080882

86. Grams ME, Sang Y, Coresh J, Ballew S, Matsushita K, Molnar MZ, et al. Acute kidney injury after major surgery: a retrospective analysis of veterans health administration data. Am J Kidney Dis. (2016) 67:87280. doi: 10.1053/j.ajkd.2015.07.022

87. Walther CP, Winkelmayer WC, Niu J, Cheema FH, Nair AP, Morgan JA, et al. Acute kidney injury with ventricular assist device placement: national estimates of trends and outcomes. Am J Kidney Dis. (2019) 74:6508. doi: 10.1053/j.ajkd.2019.03.423

88. Hughes JT, Majoni SW, Barzi F, Harris TM, Signal S, Lowah G, et al. Incident haemodialysis and outcomes in the Top End of Australia. Aust Health Rev. (2020) 44:234-40. doi: 10.1071/AH18230

89. Sud M, Tangri N, Pintilie M, Levey AS, Naimark DM. Progression to Stage 4 chronic kidney disease and death, acute kidney injury and hospitalization risk: a retrospective cohort study. Nephrol Dial Transplant. (2016) 31:112230. doi: 10.1093/ndt/gfv389

90. Siew ED, Parr SK, Abdel-Kader K, Eden SK, Peterson JF, Bansal N, et al. Predictors of recurrent AKI. J Am Soc Nephrol. (2016) 27:1190200. doi: 10.1681/ASN.2014121218

91. Grosjean F, Tonani M, Maccarrone R, Cerra C, Spaltini F, De Silvestri A, et al. Under-recognized post-stroke acute kidney injury: risk factors and relevance for stroke outcome of a frequent comorbidity. Int Urol Nephrol. (2019) 51:1597-604. doi: 10.1007/s11255-019-02203-4

92. Duffy CR, Huang Y, Andrikopoulou M, Stern-Ascher CN, Wright JD, Goffman D, et al. Clindamycin, gentamicin, and risk of clostridium difficile infection and acute kidney injury during delivery hospitalizations. Obstet Gynecol. (2020) 135:59-67. doi: 10.1097/AOG.0000000000003568

93. Downes KJ, Cowden C, Laskin BL, Huang YS, Gong W, Bryan M, et al. Association of acute kidney injury with concomitant vancomycin and Piperacillin/Tazobactam treatment among hospitalized children. JAMA Pediatr. (2017) 171:e173219. doi: 10.1001/jamapediatrics.2017.3219

94. Nelson DA, Marks ES, Deuster PA, O'Connor FG, Kurina LM. Association of nonsteroidal anti-inflammatory drug prescriptions with kidney disease among active young and middle-aged adults. JAMA Netw. (2019) 2:e187896. doi: 10.1001/jamanetworkopen.2018.7896

95. Ekezue BF, Sridhar G, Forshee RA, Izurieta HS, Selvam N, Mintz PD, et al. Occurrence of acute renal failure on the same day as immune globulin product administrations during 2008 to 2014. Transfusion. (2017) 57:297786. doi: $10.1111 /$ trf. 14330

96. Tonelli M, Lloyd AM, Bello AK, James MT, Klarenbach SW, McAlister FA, et al. Statin use and the risk of acute kidney injury in older adults. BMC Nephrol. (2019) 20:103. doi: 10.1186/s12882-019-1280-7

97. Renoux C, Lix LM, Patenaude V, Bresee LC, Paterson JM, Lafrance JP, et al. Serotonin-norepinephrine reuptake inhibitors and the risk of AKI: a cohort study of eight administrative databases and meta-analysis. Clin J Am Soc Nephrol. (2015) 10:1716-22. doi: 10.2215/CJN.11271114

98. Goswami E, Ogden RK, Bennett WE, Goldstein SL, Hackbarth R, Somers MJG, et al. Evidence-based development of a nephrotoxic medication list to screen for acute kidney injury risk in hospitalized children. Am J Health-Syst Pharm. (2019) 76:1869-74. doi: 10.1093/ajhp/ zXz203

99. Hsu CY, Hsu RK, Yang J, Ordonez JD, Zheng S, Go AS. Elevated BP after AKI. J Am Soc Nephrol. (2016) 27:914-23. doi: 10.1681/ASN.2014111114

100. Ingelfinger JR, Kalantar-Zadeh K, Schaefer F. Averting the legacy of kidney disease-focus on childhood. Am J Nephrol. (2016) 43:5864. doi: 10.1159/000444509

101. Robinson C, Jeyakumar N, Luo B, Wald R, Garg A, Nash D, et al. Longterm kidney outcomes following childhood acute kidney injury receiving dialysis: a population-based cohort study. J Am Soc Nephrol. (2021) 32:200519. doi: 10.1681/ASN.2020111665

102. Madsen NL, Goldstein SL, Froslev T, Christiansen CF, Olsen M. Cardiac surgery in patients with congenital heart disease is associated with acute kidney injury and the risk of chronic kidney disease. Kidney Int. (2017) 92:751-6. doi: 10.1016/j.kint.2017.02.021

103. Parikh CR, Greenberg JH, McArthur E, Thiessen-Philbrook H, Everett AD, Wald $\mathrm{R}$, et al. Incidence of ESKD and mortality among children with congenital heart disease after cardiac surgery. Clin J Am Soc Nephrol. (2019) 14:1450-7. doi: 10.2215/CJN.00690119

104. Hou SH, Bushinsky DA, Wish JB, Cohen JJ, Harrington JT. Hospitalacquired renal insufficiency: a prospective study. Am J Med. (1983). 74(2):243-8.

105. Nash K, Hafeez A, Hou S. Hospital-acquired renal insufficiency. Am J Kidney Dis. (2002). 39(5):930-6.

106. Goldstein SL, Kirkendall E, Nguyen H, Schaffzin JK, Bucuvalas J, Bracke T, et al. Electronic health record identification of nephrotoxin exposure and associated acute kidney injury. Pediatrics. (2013). 132:e756-67.

Conflict of Interest: The authors declare that the research was conducted in the absence of any commercial or financial relationships that could be construed as a potential conflict of interest.

Publisher's Note: All claims expressed in this article are solely those of the authors and do not necessarily represent those of their affiliated organizations, or those of the publisher, the editors and the reviewers. Any product that may be evaluated in this article, or claim that may be made by its manufacturer, is not guaranteed or endorsed by the publisher.

Copyright (c) 2021 Ulrich, So, Zappitelli and Chanchlani. This is an open-access article distributed under the terms of the Creative Commons Attribution License (CC $B Y)$. The use, distribution or reproduction in other forums is permitted, provided the original author(s) and the copyright owner(s) are credited and that the original publication in this journal is cited, in accordance with accepted academic practice. No use, distribution or reproduction is permitted which does not comply with these terms. 